\title{
DOSSIÊE
}

\section{Autopoiesis, la unidad de una diferencia: Luhmann y Maturana}

DARÍO RODRÍGUEZ M.*, JAVIER TORRES N.**

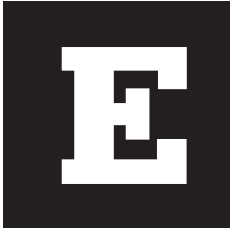

I trabajo al que Niklas Luhmann dedicó generosamente su vida dejó definitivamente instalada, en el cuerpo teórico de la sociología, una serie de conceptos cuyo origen proviene de la biología empírica. En efecto, el término de autopoiesis y sus conceptos relacionados fueron acuñados originalmente por el biólogo Humberto Maturana que requería de un aparato conceptual que le permitiera explicar la organización de los seres vivos. La incorporación de estos conceptos a la teoría sociológica implicó, necesariamente, su apropiación en los términos de un marco conceptual que Niklas Luhmann venía desarrollando por espacio de veinte años. Con ello, el complejo conceptual proveniente de la biología adquirió su dimensión propiamente sociológica, razón por la cual no se puede hablar de una biologización de la sociología (Lipp, 1987), ni tampoco de la simple adopción metafórica de conceptos provenientes de una disciplina diversa (Rodríguez, 1987).

En el presente artículo intentaremos presentar los principales elementos de la teoría luhmanniana, el desarrollo conceptual de Humberto Maturana y su recepción en el marco que Luhmann venía construyendo.

\footnotetext{
* Professor do Instituto de Sociología, Pontificia Universidad Católica de Chile. Dr.

** Professor do Departamento de Desarrollo Humano, Universidad Iberoamericana. Dr.
} 
La obra del profesor Luhmann consiste en la elaboración de una superteoría, con pretensiones de universalidad, vale decir, que reclama aplicabilidad para todo fenómeno social. Esta pretensión de universalidad no ha de ser entendida, no obstante, como un intento de excluir otras posibles interpretaciones teóricas que - desde la misma sociología o desde otras disciplinas; desde la ciencia o desde otros subsistemas de la sociedad - puedan levantarse alternativamente. Por el contrario, la teoría de sistemas de Niklas Luhmann se ha construido en un diálogo constante con diferentes esfuerzos conceptualizadores provenientes de la filosofía, la sociología, la lógica formal, el derecho, la teología, la biología, la física, etc. En este intercambio se desarrolló un marco conceptual que, manteniendo un hilo central que guió la investigación desde sus comienzos hasta su estado definitivo, incorporó elementos de diversas procedencias. Así, la teoría resultante ofrece una amplia variedad conceptual que le permite dar cuenta de los fenómenos sociales de manera radicalmente novedosa y que facilita, además, el diálogo interdisciplinario.

Creemos poder decir sin exageración que la obra de Luhmann se perfila como el trabajo teórico de mayor envergadura que haya sido elaborado en la sociología del siglo XX. Por otra parte, al incluir elementos de otras áreas del saber, tales como la cibernética, la biología, la matemática, etc., ha permitido por vez primera observar el fenómeno social desde la perspectiva de su creación en el acto mismo de conocer, superando así la vieja dicotomía entre sujeto y objeto. En esta propuesta el sujeto es reemplazado por el observador y el objeto por lo observado, pero no se trata ya más de un observador que - como una placa fotográfica - limita su acción a la mera pasividad, al simple hecho de dejarse impresionar por el objeto observado. El observador reconoce en esta teoría una posición más activa, conoce mediante esquemas de distinción, que ha incorporado autorreferencialmente y que le permiten establecer 
diferencias recibiendo, por tanto, noticias de diferencia. Observador y observado quedan, por consiguiente, integrados en el acto creativo del conocimiento, donde - sin embargo - permanece un punto ciego inmanente a toda observación: los esquemas de distinción utilizados por el observador. Estos esquemas de distinción pueden ser, a su vez, vistos por el observador de segundo orden, aquel que observa a un observador observando, pero - nuevamente - este observador de segundo orden no puede ser testigo de sus propios esquemas de distinción. No existe, por tanto, un super observador científico poseedor de la verdad absoluta y de allí se desprende que la teoría de sistemas de Niklas Luhmann tenga la pretensión de ser aplicable a cualquier fenómeno social, pero que no pueda pretender la exclusividad ni reclamar para sí la posición única de observador último, dueño de la verdad definitiva.

A continuación trataremos de presentar un breve esbozo de algunos momentos de la evolución de la teoría, con el objeto de mostrar que ésta, a pesar de haber ido introduciendo, a lo largo de los años, conceptos y terminologías de fuentes no estrictamente ancladas en la tradición sociológica, ha consistido fundamentalmente en el desarrollo del pensamiento que puede encontrarse bosquejado en forma programática en los primeros ensayos de este autor, que datan de comienzos de la década de los sesenta (Luhmann, 1962).

Teniendo en vistas la necesidad de elaborar un marco conceptual adecuado para el estudio de la sociedad, Luhmann se encontró con que la teoría de sistemas en boga era insuficiente para una tarea de tal complejidad. El esfuerzo señero de Parsons - de intentar comprender a la sociedad como un sistema abierto y, al mismo tiempo, como el sistema de los sistemas -, resultaba contradictorio e insatisfactorio. Tampoco bastaba con recurrir a simples analogías cibernéticas u orgánicas, que demostraban ser incapaces de entender la particularidad de sistemas constituyentes de sentido. El antiguo modelo - de la filosofía práctica véteroeuropea - consistente en 
considerar una parte: el subsistema político, por el todo: la sociedad, no era tampoco una alternativa promisoria (Luhmann, 1971, p. 24). La conclusión, por consiguiente, es que la teoría de sistemas no estaba lo suficientemente desarrollada para enfrentar con ella a sistemas de sentido que, tales como los sistemas sociales, ofrecían una complejidad mayor que la que podía absorber el instrumental teórico disponible. Se hacía necesario, por lo tanto, construir la teoría de sistemas sociales - inspirándose en los más modernos avances conceptuales en distintas disciplinas - para luego aproximarse con su ayuda a la sociedad moderna y sus subsistemas funcionales.

En su estudio acerca de las posibilidades ofrecidas por la teoría de sistemas, Luhmann pronto encontró que las investigaciones relacionadas con el paradigma de la autorreferencia contenían elementos que podrían ser de utilidad en la comprensión de sistemas como los sociales. El trabajo de von Foerster respecto a la autoorganización y el de Spencer-Brown que formaliza la autorreferencia son incluidos muy temprano entre los instrumentos de la teoría luhmanniana. A comienzos de la década de los ochenta, Luhmann había ya publicado aproximadamente 25 libros y 150 artículos (Luhmann y Torres, 1996, p. 275-300). Su teoría era considerada una de las más importantes propuestas de la segunda mitad del siglo veinte y su polémica con Habermas continuaba a través de los artículos que cada uno de ellos publicaba cada año. Es precisamente en los marcos de esta polémica y motivado por una crítica al libro de Habermas: Theorie des kommunikativen Handelns que en 1982 Luhmann publica Autopoiesis, Handlung und kommunikative Verständigung, trabajo en el que hace uso por vez primera del concepto de autopoiesis acuñado por Humberto Maturana. En lo que sigue intentaremos hacer una breve reseña de la obra de Maturana, con el objeto de hacer más clara la importancia de la decisión de Luhmann de incorporar sus conceptos a su teoría de los sistemas sociales. 
La teoría general de sistemas encuentra en el significativo trabajo del biólogo chileno Humberto Maturana Romecín, la posibilidad de cumplir con el sueño de von Bertalanffy: Ilegar a elaborar un aparataje conceptual lo suficientemente poderoso como para poder permitir la comunicación interdisciplinaria, transformándose en un lenguaje universal para la ciencia.

Maturana ha desarrollado una biología del conocimiento, que parte de la constatación empírica de la imposibilidad de distinguir, en la experiencia, entre ilusión y percepción. Dada esta condición, carece de fundamento pretender apoyarse en el objeto externo como factor de validación del conocimiento científico, por lo que Maturana (1996, p. 30) afirma: las explicaciones científicas no explican un mundo independiente, explican la experiencia del observador.

Ante esta constatación, Maturana emprende un ambicioso proyecto teórico, que lo lleva a replantear los fundamentos del conocimiento humano y, como consecuencia, a diseñar una nueva base para el método científico. El criterio de validación de las explicaciones científicas ya no necesita recurrir a la realidad objetiva, sino reconocer que su fuente se encuentra en la experiencia: en la ciencia no se explica el mundo, sino la experiencia.

Maturana es el pensador que hoy en día ha dado con el principio teórico más radical para entender un gran número de esferas y problemas de la sociedad contemporánea: el "material explosivo" de la autopoiesis (Luhmann, 1991, p. 480). ${ }^{1}$ La radicalidad explosiva del concepto de autopoiesis consiste en que obliga a mirar desde otra perspectiva un número elevado de certezas con las que operamos. El beneficio de esta perspectiva consiste precisamente en que es otra y no tanto en el juicio de valor (que sería todavía prematuro) de que es mejor...

Maturana es, antes que otra cosa, un biólogo. Sin embargo, el principio teórico con el que aborda la explicación de la reproducción de la vida, 
ha sido asumido (con suficiente reespecificación) en muchas campos de lo que la tradición ha venido llamando ciencias del hombre. Con el concepto de autopoiesis tenemos uno de esos casos claros en que se confirma la esperanza de escaparse de la contraposición entre ciencias de la naturaleza (duras) y ciencias del espíritu (blandas); o también entre ámbitos de objetos que obedecen a leyes y ámbitos objetuales que sólo pueden ser interpretados en forma de textos.

El resultado de las deliberaciones teóricas que propone Maturana revientan el formato comparativamente modesto de una teoría universal circunscrita al campo de la disciplina biológica. Esta teoría, cuyo gozne está constituido por la noción de autopoiesis, no es en sentido estricto biología, sino habría que compararla con esos diseños metateóricos que cumplen con la función de servir de cosmovisiones.

La imagen del mundo que aporta Maturana es intensamente translúcida: el principio constitutivo de la célula, en calidad de ultraelemento de los organismos, se mantiene en todos los niveles de complejidad que tengan que ver con lo vivo: células, organismos, sistema nervioso, comunicación, lenguaje, conciencia, sociedad. Con otras palabras: no hay discontinuidad entre lo social, lo humano y sus raíces biológicas. Esta base, contrariamente a muchas de las consecuencias que se derivaron de la teoría de la evolución de Darwin, no expresa preferencias de dominio del más apto:

elamor, o si no queremos usar una palabra tan fuerte, la aceptación del otro junto a uno en la convivencia, es el fundamento biológico del fenómeno social; sin amor, sin aceptación del otro junto a uno no hay socialización y sin socialización no hay humanidad. Cualquier cosa que destruya o limite la aceptación del otro junto a uno, desde la competencia hasta la posesión de la verdad, pasando por la certidumbre ideológica, destruye o limita el que se dé el fenómeno social, y por tanto lo humano, porque destruye el proceso biológico que lo genera 
Sociologias, Porto Alegre, ano 5, no 9, jan/jun 2003, p. 106-140

(Maturana y Varela, 1984, p. 163).

\section{Autopoiesis}

Si se observa la evolución de la materia en nuestro planeta (situada aproximadamente hace cinco mil millones de años) hay un suceso que permite imaginar el surgimiento de lo vivo. No todo intercambio molecular condujo a la aparición de lo que hoy se designa como viviente. Las moléculas que forman láminas de mica no tuvieron las propiedades necesarias para permitir en ellas el surgimiento de la células. Fue menester contar con moléculas capaces de clausurarse frente al medio ambiente. Esto fue posible gracias a la generación de membranas suficientemente estables y, a la vez, plásticas. Este tipo de moléculas (y sólo éste) fue capaz de formar barreras efectivas $y$, al mismo tiempo, tener propiedades cambiantes para difundir iones en tiempos relativamente largos, con respecto a las velocidades moleculares. A este tipo de moléculas pertenecen las proteínas cuya flexibilidad y capacidad de asociación es prácticamente ilimitada. Sólo cuando en la historia del planeta tierra se dieron las condiciones para la formación de este tipo de proteínas surgió la vida.

El punto decisivo que permite trazar un límite entre la continua producción molecular abiógena (todavía sin la característica de lo vivo), que se llevaba a cabo en la superficie de los mares y en la atmósfera y la irrupción de lo vivo, estriba en que hubo un momento en el que fue posible la formación de cadenas de reacciones moleculares de un tipo peculiar. Esta peculiaridad Maturana la llama autopoiesis. La noción de autopoiesis sirve para describir un fenómeno radicalmente circular: las moléculas orgánicas forman redes de reacciones que producen a las mismas moléculas de las que están integradas. Tales redes e interacciones moleculares que se producen a sí mismas y especifican sus propios límites son los seres vivos. Los seres vivos, entonces, quedan definidos como aquellos cuyas 
característica es que se producen a sí mismos, lo que se indica al designar la organización que los define, como organización autopoiética: La característica más peculiar de un sistema autopoiético es que se levanta por sus propios cordones y se constituye como distinto del medio circundante a través de su propia dinámica, de tal manera que ambas cosas son inseparables (Ibid, p. 28).

Cinco son, por lo menos, las propiedades que caracterizan el fenómeno autopoiético:

Autonomía: la célula pone de manifiesto la superación de la correspondencia punto por punto con respecto al medio ambiente. Ya no es la célula un componente constituido sólo de átomos o moléculas, sino una forma específica (autopoiética) de combinación de dichos componentes. Esta forma-específica-de-combinación exige una perspectiva de autonomía en el sentido de que la célula requiere de la creación de distancia con respecto al medio circundante. La autonomía de lo orgánico, en último término, significa que sólo desde la perspectiva de la célula se puede determinar lo que le es relevante $y$, sobre todo, lo que le es indiferente: Así, en el presente de esta deriva natural celular, las membranas operan transportando iones de sodio y calcio, y no otros (Ibid, p. 51).

Emergencia: el surgimiento del orden cualitativo de la célula - diverso, por ejemplo, a las moléculas que forman láminas de mica - no puede concluirse a partir de las características materiales o energéticas que, a su vez, componen la célula. La emergencia señala precisamente la irrupción de un nuevo orden, cuyas características sólo pueden ser inducidas una vez que el nuevo orden ya está constituido. Lo que es emergente en la célula no es que las moléculas puedan crear indefinidamente más moléculas, sin ninguna necesidad de recurrir a elementos energéticos del medio ambiente, sino al hecho de que las células dependen, en su operación, de la forma en que están organizadas y de cómo esta organización se lleva a efecto.

Siempre que se habla de un orden emergente, queda presupuesto 
(pero no incluido) el continuo de energía o de materialidad sobre el que una unidad se sostiene. La emergencia del orden modifica la composición interna de la materia: por ejemplo, la electrónica interna del átomo se transforma en cuanto surge el orden emergente de las macromoléculas. De aquí, que la energía atómica no forme parte del proceso químico que constituye la célula (ipor suerte!). Si se empleara un diseño de teoría insólito como el de la teoría de sistemas que se orienta por la distinción sistema/entorno, entonces se tendrían que considerar a los átomos como entorno del sistema de organización autopoiética de la célula.

Clausura operativa: Los sistemas autopoiéticos son sistemas cuya operación es cerrada y cuyos componentes son producidos al interior de un proceso recursivo que se lleva a cabo dentro de una retícula clausurada. Cerradura no deberá entenderse aquí como lo opuesto a apertura, sino como la condición de su posibilidad. Lo que está clausurado en la autopoiesis es el control mismo mediante el cual los elementos se organizan de manera emergente. Este control-mismo-de-la-organización se puede visualizar en la siguiente cadena de la evolución: átomos que se transforman en moléculas; moléculas inorgánicas que se convierten en cristales y soluciones; macromoléculas (es decir moléculas polímeros) que pasan a ser células, células que se transforman en organismos multicelulares.

La clausura operativa de la autopoiesis hace relación directa al nivel de estabilidad que alcanza una operación, bajo condiciones determinadas, y en la que necesariamente esta operación tiende a formar un cálculo recursivo que siempre debe volver sobre sí mismo (autorreferente).

Con la caracterización de clausura operativa lo que se pretende establecer es que las células producen operaciones exclusivas que reproducen la vida que las mantiene en vida. Esto quiere decir: el sistema sólo puede disponer de sus propias operaciones; o, con otras palabras, dentro del sistema no existe otra cosa que su propia operación. Esta operación única logra conformar dentro del sistema dos acontecimientos 
fundamentales: la construcción de estructuras y la autopoiesis.

Auto construcción de estructuras: dado que la operación de la célula está clausurada, no puede importar estructuras: ella misma debe construirlas. Por tanto el concepto de autoconstrucción deberá entenderse en primera línea como producción de estructuras propias, mediante operaciones propias. Los sistemas clausurados en su operación producen sus propios elementos $y$, por consiguiente, sus propios cambios estructurales. No existe una intervención causal del entorno en el sistema sin que el mismo sistema lo provoque: todo cambio de estructuras - trátese de procesos de adaptación o de rechazo -, es en última instancia autoinducido.

Autopoiesis significa, entonces sobre todo, determinación del estado siguiente del sistema a partir de la estructuración anterior a la que llegó la operación.

En resumen, con el principio de la autopoiesis se afirman, con radicalidad, cuando menos, cinco características: autonomía, emergencia, clausura operativa, autoestructuración y reproducción autopoiética. Todo el fenómeno de la organización de lo vivo es así, entonces, un acontecimiento que puede ser explicado a la luz de esta fenomenología de cinco facetas.

La cadena evolutiva da inicio con la autopoiesis de la célula y, en un sentido estricto, se afirma que ésta es el único fenómeno biológico propiamente autopoiético. El siguiente escalón lo constituyen los organismos pluricelulares (metacelulares de segundo orden: por ejemplo, una ballena). Aunque se pudiera dudar acerca de si estos organismos complejos se tuvieran que considerar, a su vez, autopoiéticos, Maturana no deja dudas al afirmar que lo que sí poseen es clausura operaciona/en su organización: su identidad está especificada por una red de procesos dinámicos cuyos efectos no salen de esa red (Ibid, p. 59).

Si se continúa en la escala de complejidad de lo vivo, el sistema nervioso se puede explicar a la luz de este principio fenomenológico del 
estar clausurado operativamente. Por lo general, en la comprensión más usual sobre el sistema nervioso, se tiene la idea de que se trata de un instrumento que obtiene información del medio ambiente, que luego utiliza para construir una representación del mundo y, con ello, poder responder con una conducta adecuada para sobrevivir en él. Sin embargo: el sistema nervioso no "capta información" del medio ambiente, como a menudo se escucha sino que, al revés, trae un mundo a la mano al especificar qué configuraciones del medio son perturbaciones y qué cambios gatillan éstas en el organismo (Ibid, p. 113).

El rendimiento evolutivo del sistema nervioso consiste en que posibilita la expansión del campo de estados posibles del organismo. De esta manera se trata de un aumento de configuración de los estados internos que, después, un observador puede enjuiciar de correlación acoplada con el mundo, pero que, en sentido restringido, no son sino formas cristalizadas del modo interno de operación de un sistema.

Debido a la característica expansiva de comportamiento interior que se logra mediante el sistema nervioso, el fenómeno del conocer no es exclusivo del ser humano. En el plano de la organización de lo viviente todo operar orgánico es conocimiento. Todo hacer es conocer, reza el adagio de Maturana. El conocimiento no opera - y no puede operar - valiéndose de una representación que se hace sobre el medio ambiente. Conocer es el operar de los componentes de un sistema dentro del dominio de sus estados internos y de sus cambios estructurales.

En la lógica de este principio de clausura, la evolución desarrolla, en primera línea, unidades orgánicas individuales que operan con autonomía. Las vinculaciones colectivas surgen en el momento en que las interacciones entre los organismos de una misma especie, a lo largo de una historia, adquieren un carácter de recurrencia. De tal manera, que se puede afirmar que estos organismos quedan acoplados en el plano de la estructura, lo que, a su vez, permite la conservación de su individualidad autopoiética en la 
larga historia de sus interacciones. Lo colectivo, entonces, no es un fenómeno esencialmente humano sino biológico: a partir de unidades individuales autónomas surge un orden que coordina el comportamiento operativo de las unidades individuales orgánicas, cuando entran en relaciones recurrentes. El sustento biológico de la vida no contrapone individuo y colectividad: se es altruistamente egoísta y egoístamente altruista, porque la realización individual incluye la pertenencia al grupo que integra (lbid, p. 131).

La comunicación, en su sentido más general, es la coordinación de conductas que, por sí mismas, no podrían crear actos colectivos recurrentes. De aquí que la comunicación no transfiera contenidos, sino más bien coordine comportamientos: hay comunicación cada vez que hay coordinación conductual en un dominio de acoplamiento estructural(lbid, p. 130).

Aunque la comunicación no se agota con la aparición de las conductas lingüísticas, es evidente que el lenguaje es un fenómeno inédito, por el significado inmensamente abarcador para el ser humano; aunque haya, en otros niveles del orden del mundo animal, equivalentes de comportamientos lingüísticos.

El lenguaje introduce una doble dimensión en la historia evolutiva del ser humano: primero, por el lenguaje emerge la experiencia de lo mental y la conciencia humana como expresión del centro más íntimo del hombre; segundo - y en esto quizás consista lo más sorprendente -, es que al situar al individuo en el plano de la coordinación de las interacciones recurrentes junto a otros, despoja al individuo de toda certidumbre absoluta de lo personal y lo invita a situarse en una perspectiva más amplia: la de la creación de un mundo junto con otros (Ibid, p. 163).

Autopoiesis y teoría del conocimiento

Si estos presupuestos teóricos se trasladan a la teoría del conocimiento producen impactos cuyos efectos son incalculables, ya que inducen a pensar que el conocimiento sólo es posible en la medida en que está sustentado en 
operaciones que no pueden entablar ningún contacto con el entorno.

La teoría del conocimiento ha discurrido - expuesto de manera tosca, sobre todo si se tiene en cuenta el alto nivel técnico alcanzado en la discusión - sobre dos ejes que han sido, hasta ahora, irreconciliables.

El primero, el racionalismo, sostiene que el conocimiento no puede partir de la inmediatez de la realidad, sino exclusivamente de la posibilidad escueta. Posibilidad significa simplemente inteligibilidad exenta de paradojas. De aquí que el conocimiento sea un proceso eminentemente deductivo que se desprende de conceptos primeros y axiomas, con tal de que se cuide de no caer en contradicción.

De manera radicalmente opuesta se encuentra el empirismo que opera bajo el presupuesto de que es la realidad la que ha de decidir lo que es verdadero o lo que es falso. Los hechos son datos en bruto que pueden ser aclarados, con la única condición de que se emplee un método experimental lo suficientemente riguroso. De esta manera, la realidad misma es la que confirma y la que permite descartar errores. El empirismo sostiene, entonces, de muchas maneras y muy complejas, que existe una certeza inmediata del mundo exterior y que ella es lo confirmante.

Kant - y probablemente desde la perspectiva especializada de la epistemología constituya la cumbre - en contra del racionalismo esta convencido de que los juicios universales y necesarios (todos los hombres son mortales) no pueden ser solamente analíticos, sino también sintéticos: no sólo han de tener capacidad de explicar, sino la de ampliar el contenido inmediato de la realidad.

Con todo, estos juicios sintéticos que han de tener validez universal y necesaria, no pueden fundarse en la experiencia en el sentido del empirismo - puesto que ésta aporta sólo lo singular y lo contingente.

El problema para Kant se resuelve si es que existen juicios sintéticos a priori, que se fundamentan en principios preexperimentales y que, a pesar de eso, aportan un paso adelante al conocimiento. Este planteo sitúa el 
problema de la estructura del conocimiento con una profundidad que sobrepasa las representaciones epistemológicas de su época. Desde entonces, se va a constituir en tradición comprender el conocimiento a partir de las condiciones previas de su posibilidad: nuestra manera de conocer los objetos, en cuanto ésta es posible, a priori (Kant). La tesis final de descarga dice: la realidad en síes irreconocible.

Debe quedar abierto, para fines de este artículo, si esta caracterización compacta corresponda plenamente a los esfuerzos teóricos de Kant.

Maturana resume este recorrido clásico de la teoría cognitiva, en términos de trampas del conocimiento. La primera trampa es creer que el mundo de los objetos puede dar instrucciones al conocimiento, cuando de hecho, no hay un mecanismo que permita tal información. La segunda, es que una vez que no existe el control de la certeza inmediata, abandonados a la oscura interioridad de lo posible pensado, amenaza el caos y la arbitrariedad.

La primera trampa cree que el sistema nervioso trabaja con representaciones del mundo, cuando de hecho su modo de operar está determinado, de momento a momento, desde el interior de la clausura operacional. La segunda, tiende a atribuir a la clausura de operación una absoluta soledad cognoscitiva (solipsismo), y se desentiende de explicar la asombrosa conmensurabilidad entre el operar del organismo y el mundo: La solución, como todas las soluciones de aparente contradicciones, consiste en salirse del plano de la oposición y cambiar la naturaleza de la pregunta a un contexto más abarcador (Ibid, p. 89).

Maturana sugiere caminar en el filo de la navaja y llevar una contabilidad lógica para encontrar salida al problema del conocimiento. Propone una distinción: la de la operación/la de la observación. Desde la operación, el conocimiento está clausurado y sólo responde desde sus interiores determinaciones estructurales. El observador, que está colocado 
fuera de la operación y que mira desde un plano más abarcador, puede llevar a efecto enlaces causales entre operación y mundo circundante que no son accesibles (como observaciones conscientes, por ejemplo) a los organismos que los efectúan:

al mantener limpia nuestra contabilidad lógica, esta complicación se disipa, ya que nos hacemos cargo de estas dos perspectivas y las relacionamos en un dominio más abarcador que nosotros establecemos. Así, no necesitamos recurrir a las representaciones, ni necesitamos negar que el sistema opera en un medio que le es conmensurable como resultado de una historia de acoplamiento estructural (Ibid, p. 91).

Si estas reflexiones se llevan al plano formal de la generalización, el que el conocimiento esté constituido por una operación que está clausurada quiere decir que no puede establecer ningún contacto con el entorno. Este es un principio teórico complicado que contradice toda la tradición reflexiva sobre el conocimiento y que debe quedar, aquí, consignado como llamada de atención.

Todo conocimiento sobre la realidad debe realizarse como actividad interna del conocimiento, dirigida mediante distinciones propias (para las cuales no existe ninguna correspondencia con el entorno).

La pregunta horadante es entonces icómo se configura el conocimiento? Toda la teoría del conocimiento depende de la respuesta que se dé a este planteo. Maturana para este problema difícil propone un concepto, igualmente, difícil: acoplamiento estructural. Esta noción presupone que todo conocimiento (que es una operación emergente autopoiética) opera como un sistema determinado sólo desde el interior mediante sus propias estructuras. Se excluye, entonces, el que datos existentes en el entorno puedan especificar, conforme a las estructuras internas, lo que sucede en el sistema. 
Maturana diría que el acoplamiento estructural se encuentra de modo ortogonal con respecto a la autodeterminación del sistema. Lo que quiere decir que una certeza inmediata de la realidad, aunque no determina lo que sucede en el conocimiento, debe estar presupuesta, ya que de otra manera la autopoiesis cesaría. En este sentido todo conocimiento está previamente acoplado de manera flexible al entorno (o no existiría), pero hacia el interior del radio de acción que se le confiere, el conocimiento tiende a dar respuestas acopladas en modo rígido. La realidad, por consiguiente, sirve sólo de medio flexible y abierto, para que el conocimiento aporte, desde sí mismo, acoplamientos rígidos y configurados según su propia idea de orden...

Autopoiesis y teoría de la sociedad

Cuando un concepto, por su riqueza de aplicación, se impone con plausibilidad más allá del contexto de inicio en el que fue pensado, se transforma en una estructura general que puede ser aplicada en muchos campos: por ejemplo, la categoría de proceso fue descubierta, primero, en la jurisprudencia y luego adaptada a la química. Este tipo de nociones evaden los controles de origen y dan pie a interpretaciones semánticas inesperadas, a las que, en ocasiones, sus mismos creadores se resisten.

Einstein aportó, más que ninguno otro, todos los presupuestos para el desarrollo de la teoría cuántica, pero mantuvo serias reservas para aceptarla. El resultado ha sido que la física debe orientarse por dos teorías universales que, hasta ahora, no ha sido posible conciliar: la teoría de la relatividad general que parece gobernar la estructura a gran escala del universo y la mecánica cuántica, cuyo foco de atención se dirige a la estructura de micro escala en lo molecular.

Con la noción de autopoiesis ha acontecido un fenómeno, si no exactamente igual, sí, al menos, parecido: al quedar expuesta al proceso de reespecificación en cada una de las disciplinas del espíritu, ha tenido 
que sufrir modificaciones en la interpretación. La diferencia más notable de algunas precisiones de sentido de este concepto se localiza en la discusión actual entre biología y sociología.

Este aviso de ninguna manera pretende expresar un juicio negativo sobre Maturana. Al contrario, esto es sólo el reflejo de la vehemencia y el fenómeno expansivo que ha provocado su teoría.

Para resaltar con más intensidad la importancia de la obra de este biólogo chileno es conveniente prestar atención a la manera en que el destacado sociólogo Niklas Luhmann desarrolla la teoría de la autopoiesis de la sociedad.

Así como el origen de la vida tiene que ver con el proceso de clausura de ciertas proteínas, así, en la propuesta de Luhmann, aquello que se ha designado como proceso de humanización (socialización) fue posible gracias a que surgió una forma emergente, una red cerrada (autopoiética) de comunicación.

Sólo a esta red cerrada de comunicación es posible designar con el concepto de sociedad. Fuera de esta red no existe comunicación. Ella es la única que utiliza este tipo de operación y en esta medida es real y necesariamente cerrada.

Desde el momento en el que en la humanidad da comienzo el proceso civilizatorio (Norbert Elias), no importa la fecha en que esto se precise (i4,4 millones de años?), la sociedad es una forma clausurada de comunicación que tiene la cualidad de albergar dentro de sí misma, de manera omniabarcadora, todo lo que tenga que ver con formas de comunicación de sentido.

La evolución encontró en los procesos comunicacionales el medio de la socialización de los seres humanos. En otras palabras, la civilización y sus resultados son consecuencia de las condiciones del cometido de la comunicación. No son los seres humanos los creadores del proceso de su propia civilización, al contrario: los seres humanos se hacen dependientes 
de esta red emergente de orden superior, bajo cuyas condiciones pueden elegir los contactos con otros seres humanos. Esta red de comunicación de orden superior es lo que denominamos sociedad. Lo social no surge del hombre. Consiste en una solución de tipo evolutivo que precede a los sujetos que está encaminada a proveer de estructuras (iformas!) de sentido que se imponen a la tendencia radical de la desintegración.

La socialización de los seres humanos no es, en sentido estricto, humanización. Si se parte de la premisa de que la sociedad es pura comunicación, el desarrollo de lo social se debe entender como un aumento en el desempeño comunicativo, pero no como una ampliación de humanización en la dirección de Rousseau o de Nietzsche (el primero, la perfectibilidad de la naturaleza humana; el otro, la superación de las energías dionisíacas).

Lo social nunca ha sido (y probablemente nunca lo será) el espacio de la realización absoluta de las posibilidades más humanas del hombre. La sociedad manifiesta una consistencia propia (si bien dinámica y evolutiva), una regulación autorreferente que da pie a que cada individuo la experimente en grados de profundidad (o de decepción) y en direcciones diversas. Pero estos grados de vivencia subjetivo no pertenecen propiamente al ámbito de lo social: están ubicados en el otro lado de la forma de lo social, en el entorno. El descubrimiento moderno de lo inconmensurable de la interioridad humana, a partir de Freud, advierte que no es posible construir una sociedad que pueda corresponder a tales posibilidades de variación.

Luhmann conecta directamente con el concepto de autopoiesis en el momento en que considera la sociedad como una red cerrada, autorreferente. La crítica a este tipo de sociología es el reparo de que considera la sociedad como una especie de realidad orgánica aprehensible en formato grande. Se acusa a la teoría de sociobiología. Luhmann defiende: 
Sociologias, Porto Alegre, ano 5, no 9, jan/jun 2003, p. 106-140

vida (y para Maturana no sólo describe, sino que define el concepto mismo de la vida) es aceptable para los biólogos, no se sigue de allí que el concepto sea sólo biológico. Si encontramos que los automóviles trabajan con un motor interior, esto no significa que el concepto de motor debe quedar reducido a los automóviles. Nada hay que impida el que tratemos de ver si los sistemas sociales son autopoiéticos en términos de su propio modo de producción y reproducción, en lugar de considerarlos en términos de la operación bioquímica de la vida (Sciulli, 1994, p. 42).

No sólo están organizados autopoiéticamente las unidades orgánicas, sino también las formas sociales y las conciencias de los individuos. Luhmann generaliza el concepto de autopoiesis y lo aplica a otros ámbitos de la realidad.

Los sistemas vivos, los neuronales, las conciencias, y los sistemas sociales son (para Luhmann) sistemas autopoiéticos, esto es, sistemas que se llevan a cabo gracias a una reproducción recursiva de sus elementos como unidades autónomas. El concepto de autopoiesis está tomado en la dirección de la autoconservación del sistema mediante la producción de sus propios elementos:

Como autopoiéticos nosotros queremos designar aquel tipo de unidades que producen y reproducen los elementos de los que están constituidos, a partir de los elementos de los que están constituidos. Todo lo que estos elementos utilizan como unidad (ya se trate de elementos, de procesos, de estructuras, de sí mismos) deben ser producidos mediante esas mismas unidades. O dicho de otro modo: no existe ninguna unidad que se desempeñe como input para el sistema; ni ningún output que sirva de unidad que no provenga del sistema. Esto no quiere decir que no haya ninguna 
relación con el entorno, pero estas relaciones se sitúan en un nivel de realidad distinto al de la autopoiesis (Luhmann, 1995, p. 56).

La sociedad es, pues, un orden emergente que se deslinda de lo específico de la vida orgánica y de la vida interior de las conciencias. El concepto de emergencia designa la irrupción de un nuevo orden de realidad que no puede ser explicado (ni reducido) en su totalidad, a partir de las características de la infraestructura sobre la que se encuentra sostenido. En el caso, por ejemplo, de la relación entre conciencia y cerebro, la conciencia está sustentada sobre procesos neuronales, pero las neuronas no producen ningún tipo de pensamiento o de representación. La frase, cuya patente se adjudica a Ernst Bloch, de que por más que nos paseáramos por las azoteas del cerebro nunca nos encontraríamos allí una idea, ilustra el contenido.

La dimensión de qué significa que la sociedad sea un orden emergente autopoiético debe ser sopesada con toda gravedad, ya que contradice toda la tradición filosófica y sociológica que se sustenta en la conceptualización del sujeto: el ser humano, en este tipo de tradición, constituye el ultraelemento de lo social. La tradición considera a los social como el sistema omniabarcador que se constituye a partir de individuos y del conjunto de sus relaciones. De igual manera esta tradición considera que los seres humanos son los que comunican y se comunican con otros.

Desde el momento en que Luhmann opta por la conceptualización de la autopoiesis rompe con la tradición del pensamiento europeo. Lo social, en esta teoría, no está constituido por lo seres humanos, sino por la comunicación. En esta dinámica de pensamiento los seres humanos no están considerados como los creadores de la comunicación. La comunicación no es ningún resultado de la acción del ser humano, sino una operación que solamente se hace posible genuinamente por sí sola, es decir, por la sociedad:

No es el hombre quien puede comunicarse, sólo la 
Sociologias, Porto Alegre, ano 5, no 9, jan/jun 2003, p. 106-140

comunicación puede comunicar. La comunicación constituye una realidad emergente sui generis. De la misma manera como los sistemas de comunicación (como también por otra parte los cerebros, las células, etcétera), los sistemas de conciencia también son sistemas operacionalmente cerrados. No pueden tener contacto unos con otros. No existe la comunicación de conciencia a conciencia, ni entre el individuo y la sociedad. Si se quiere comprender con suficiente precisión la comunicación es necesario excluir tales posibilidades (aun la que consiste en concebir la sociedad como un espíritu colectivo). Solamente una conciencia puede pensar (pero no puede pensar con pensamientos propios dentro de otra conciencia) y solamente la sociedad puede comunicar. Y en los dos casos se trata de operaciones propias de un sistema operacionalmente cerrado, determinado por la estructura (Luhmann, De Giorgi, 1993, p. 52).

La sociedad es autónoma no sólo en el plano estructural (a lo que había llegado el estructuralismo), sino también y fundamentalmente en el plano del control de la organización de sus estructuras. La sociedad puede hacer surgir operaciones propias solamente empalmándolas a operaciones propias y en anticipación a ulteriores operaciones de la sociedad.

No obstante, zestá Luhmann en lo justo? Oigamos ahora al maestro chileno:

Esta discrepancia con Luhmann no es trivial... Ciertamente se puede hacer lo que Luhmann hace al distinguir un sistema cerrado definiblemente autopoiético en el espacio de las comunicaciones que él llama sistema social. Lo que yo me pregunto es si la noción de lo social como ésta surge en el ámbito cotidiano y se aplica adecuadamente a ese sistema: es decir, me pregunto si el sistema que Luhmann distingue como 
sistema social genera los fenómenos y experiencias que en la vida cotidiana connotamos al hablar de lo social. Yo pienso que no, que no lo hace, y pienso, por lo tanto, que la noción de lo social está mal aplicada al tipo de sistemas que Luhmann Ilama "sistemas sociales"... Lo social no pertenece a la sociología, pertenece a la vida cotidiana, y la sociología sólo hace sentido como intento explicativo de la vida cotidiana, si no, es sólo literatura. Todo lo que Luhmann parece querer explicar con su teoría de los sistemas sociales separando lo humano y dejándolo como parte del entorno, y mucho más que él no puede explicar, como el origen del lenguaje, como el origen de lo humano, se puede explicar sin ese argumento (Maturana, 1992).

Pese a la opinión crítica de Maturana, que muestra parte de las diferencias entre ambos autores (Rodríguez, 1987), de los párrafos precedentes se puede desprender que Luhmann encuentra en el trabajo de Maturana los conceptos adecuados para expresar de manera precisa y elegante, vale decir sin exceso de terminología aclaratoria y redundante, las características de los sistemas sociales. No se trata, no obstante, de la simple importación a la sociología de conceptos elaborados en el seno de la teoría biológica. Para hacer una apropiación efectiva de la conceptualización, es necesario adaptarla a los sistemas sociales y determinar sus condiciones de aplicabilidad (Lipp, 1987).

Un sistema autopoiético es el que produce sus propios componentes. Es necesario, por lo tanto, reconocer los elementos que forman los sistemas sociales y que son creados por estos mismos sistemas.

Estos elementos deben ser propiamente sociales, pasajeros y permitir 
que se establezcan conexiones entre ellos, para formar una red de producción que los produzca. Luhmann considera que la comunicación cumple con estos requisitos:

a) La comunicación es social, dado que necesita de al menos dos personas para producirse;

b) La comunicación es pasajera, apenas comienza, también empieza a desvanecerse; y

c) La comunicación va dejando puentes, va tendiendo conexiones con otras comunicaciones que permiten continuar la autopoiesis del sistema social.

La comunicación no es una acción, tampoco una acción comunicativa, sino un evento diferente, que requiere de la participación de más de un actor para tener lugar. En el concepto elaborado por Luhmann, la comunicación es una síntesis de tres selecciones:

a) La selección de una información, en que Alter decide cuál de las distintas alternativas de información de que dispone va a seleccionar: qué voy a informar, qué voy a decir.

b) La selección de una expresión o acto de comunicar, en que Alter opta por una forma de expresar la información: cómo lo digo.

c) La selección de una comprensión/incomprensión, en que Ego elige una de las posibilidades de comprensión o incomprensión de lo que A/terle ha transmitido. En este acto, Ego diferencia entre la información y la expresión: qué entiendo.

Como se puede ver, este concepto de comunicación implica la participación de ambos interlocutores y no puede ser confundido con el simple acto de comunicar. Luhmann es muy enfático al respecto y es la razón para remarcarlo, al invertir el habitual esquema de la comunicación en que es Ego el que habla y Alter el que escucha. El decir que A/ter selecciona una información y una expresión o acto de comunicar, en tanto Ego selecciona una comprensión o incomprensión, está orientado a hacer 
notar que la comunicación sólo ocurre cuando Ego reacciona comprendiendo o no.

Sin embargo, dado que - para que la autopoiesis del sistema continúe - la comunicación requiere establecer vínculos con otras comunicaciones, las distintas partes de la comunicación se atribuyen como acciones a los interlocutores: se sabe quién dijo qué. Con esto, el sistema de comunicaciones genera una versión simplificada de sí mismo, entendiéndose como un sistema de acciones. La sociología ha visto sólo esta versión simplificada de los sistemas sociales y ha entendido, por eso, que la acción es el elemento de lo social.

Las comunicaciones son eventos fugaces que se conectan entre sí mediante el sentido, que es una estrategia de selección que permite distinguir entre las comunicaciones que pertenecen al sistema y lo no perteneciente a él. El sentido que se construye en la comunicación, es intersubjetivo, pero no depende de ninguno de los hablantes: Uno de los interlocutores puede darle un sentido propio a su participación, pero para que la comunicación ocurra es indispensable que el sentido sea compartido. En el medio de una disertación acerca del concepto de autopoiesis, el profesor no puede, sin más, intercalar una frase referida a lo bien que lo pasó la velada de la víspera. Los alumnos quedarían atónitos y preguntarían por la relación entre la última comunicación y el sentido de la clase.

Los sistemas sociales se encuentran clausurados operacionalmente. Las comunicaciones que constituyen sus elementos son producidas al interior del sistema y no provienen de su entorno. Pueden referirse a aspectos del entorno, pero para que estos sean participados en la comunicación deberán ser tematizados en ella. Un trueno o un ruido ensordecedor, por ejemplo, no son parte de la comunicación. Si el ruido es demasiado grande, puede obligar a los hablantes a elevar la voz. Pudiera, incluso, interrumpir la comunicación, pero no ser parte de ella, a menos que se hable de él en la comunicación: que los interlocutores decidan trasladarse a otro lugar "porque el ruido no nos deja hablar". Se sabe con Bateson, sin embargo, 
que el ruido no es el ruido; que la palabra ruido simplemente tematiza un fenómeno ambiental que permanece fuera de la comunicación.

Para que un determinado fenómeno sea tematizado en la comunicación, es necesario que consiga hacer resonancia dentro del sistema social, vale decir, que apele a alguna posibilidad del sistema que guarde sintonía con él. Se pudiera hacer la metáfora del diapasón, que vibra por simpatía, vale decir, que comienza a emitir una nota cuando una cuerda de la guitarra afinada para esa nota es pulsada y hecha sonar. El diapasón como el sistema - no necesita ser estimulado directamente, sino que resuena en concordancia, al ser estimulado o irritado por el sonido al que está afinada su estructura. Ningún otro sonido va a provocar este resultado; el diapasón es "sordo" a todo otro sonido. Los sistemas sociales cuentan también con este mecanismo de contacto con el entorno. Su estructura resuena al ser estimulada por algún fenómeno para el que esté sintonizada. El resto de los fenómenos pasa desapercibido.

A pesar de la clausura operacional y de la autonomía de los sistemas sociales con respecto a su entorno, estos se encuentran acoplados estructuralmente a los sistemas de conciencia. Los sistemas sociales están compuestos de comunicaciones que son generadas autopoiéticamente por las propias operaciones de estos sistemas. El acoplamiento estructural de los sistemas sociales con los sistemas psíquicos conduce a que la comunicación sólo puede ser estimulada o irritada por los sistemas psíquicos y no por fenómenos u objetos físicos que puedan ser tematizados en ella. En efecto, para que un determinado suceso sea tratado en la comunicación, es preciso que sea percibido por un sistema psíquico que, luego, lo transforma en la información que participará en la comunicación.

Una crítica frecuente a la teoría de Luhmann consiste en señalar que deja fuera al ser humano, dado que considera que los sistemas sociales no están compuestos de seres humanos, sino de comunicaciones. Al respecto, es conveniente tener presente, que en esta teoría se deja al ser humano 
toda su complejidad, que no queda reducida a la de un componente que debe someterse a la autopoiesis del sistema del que es elemento. Por otra parte, las comunicaciones no pueden tener lugar con prescindencia de los sistemas psíquicos a los que están estructuralmente acopladas. La comunicación constituye el nivel de emergencia de lo social, así como la célula lo es de lo biológico. No puede haber sistemas biológicos que no cuenten con moléculas o átomos, pero lo que caracteriza lo biológico - la vida - emerge en el nivel celular.

El acoplamiento estructural consiste en una adaptación permanente entre sistemas diferentes, que mantienen su especificidad; no se puede reducir un sistema social a los sistemas psíquicos ni viceversa. Los pensamientos de un sistema psíquico no son comunicaciones, sino eventos propios de la reproducción autopoiética de la psiquis, que estimulan o irritan al sistema de comunicaciones. La comunicación, por su lado, tampoco ingresa al fluir de pensamientos del sistema psíquico de Ego o de Alter. Su papel se limita a estimular, gatillar o irritar pensamientos en el sistema psíquico. Esto nos permite entender que una misma comunicación estimule pensamientos diferentes en distintos interlocutores. La comunicación no consiste en el traspaso de un determinado contenido de un emisor a un receptor, sino en la creación intersubjetiva de sentido, que delimita un sistema social.

Lo anterior quiere decir que los sistemas sociales se encuentran determinados estructuralmente; las comunicaciones se van encadenando con otras comunicaciones y los pensamientos sólo pueden desencadenar comunicaciones posibles, vale decir, consideradas en la estructura del sistema social: un interlocutor recuerda repentinamente algo y lo quiere hacer saber, pero debe antes advertir del cambio a sus oyentes porque de lo contrario su comunicación no hará sentido. Tampoco las comunicaciones pueden generar pensamientos que no sean posibles para el sistema psíquico: no se le puede enseñar a multiplicar a un niño que no sepa sumar, sino 
que hay que permitir que la estructura de su sistema psíquico abra la posibilidad de ese cambio, para - recién entonces - gatillarlo diciéndole que una multiplicación es una suma con sumandos iguales. La determinación estructural de los sistemas sociales, conduce a que su acoplamiento con los sistemas psíquicos sea condición del sistema social, pero al mismo tiempo, que los cambios de estado del sistema social sólo puedan ser especificados por su propia estructura y no por el entorno. Ningún sistema psíquico puede determinar el sentido de una comunicación; éste es intersubjetivo.

En la teoría de sistemas, según Luhmann, han tenido lugar dos cambios de paradigmas. Un cambio de paradigma ocurre cuando el nuevo paradigma es capaz de dar cuenta de todo lo que explicaba el paradigma anterior $y$, además, incorporar nuevas distinciones. El cambio de paradigmas, por consiguiente, no significa echar por tierra lo logrado con el paradigma anterior, sino construir sobre sus logros. El nuevo paradigma, por lo tanto, ha de ser capaz de explicar al paradigma que supera.

En el primer cambio de paradigmas, el antiguo paradigma de todo/ partes es reemplazado por el de sistema/ entorno. Este cambio se produce en los inicios de la moderna teoría de los sistemas. Cuando von Bertalanffy ofrece su modelo de sistemas abiertos a su entorno, supera la antigua visión de los sistemas como entidades cerradas, caracterizadas por estar compuestas por elementos y relaciones entre estos elementos que hacían que el todo fuera más que la suma de las partes. La incorporación del antiguo paradigma en la nueva conceptualización implica que las partes son vistas como diferencias sistema/entorno al interior de un sistema.

El segundo cambio de paradigmas es el paso de la teoría de sistemas abiertos a la teoría de la autorreferencia, paradigma que se comienza a perfilar tempranamente, con los trabajos de von Foerster respecto a la autoorganización y que conducen a la teoría de la autopoiesis, elaborada por Maturana y generalizada por Luhmann. El paradigma de sistema/entorno es incorporado en el paradigma de la autorreferencia, al señalarse que la 
diferencia sistema/entorno es un momento de la autorreferencia. Los sistemas autorreferentes se diferencian respecto a su entorno.

Esto nos lleva al tema de la observación, que es otro de los puntos importantes en la teoría de Luhmann. Un sistema autorreferente se puede diferenciar de su entorno al hacer uso de la diferencia sistema/entorno como orientación. La clausura autorreferencial sólo es posible en un entorno y éste es el correlato necesario de la operación autorreferencial. La observación utiliza esquemas de distinción, pero la unidad de la diferencia se constituye en el sistema observador y no en el sistema observado. La observación tiene un punto ciego que es, precisamente, el esquema de distinción utilizado para hacerla posible. En la observación de segundo orden: la observación de la observación, se puede observar un observador observando, vale decir, haciendo uso de su esquema de distinción. En esta observación de segundo orden se puede observar el esquema de distinción utilizado por el observador, pero el observador de segundo orden no puede ver su propio esquema de distinción. No existe un observador de tercer orden, dado que para él es válido lo dicho para la observación de segundo orden: que puede ver los esquemas de distinción del observador observado, pero no los suyos propios.

Los sistemas sociales son, además, sistemas autoobservadores, lo que no significa que cuenten con operaciones capaces de observarse a sí mismas en cuanto observación en curso, porque esto sería imposible. Se trata, más bien, de sistemas en los que se introduce la diferencia sistema /entorno en el sistema que se constituye por su intermedio. Esto es lo que SpencerBrown (1979), denomina re-entry. La autoobservación es un momento operativo de la autopoiesis del sistema porque la reproducción de los elementos ha de basarse en esta diferencia sistema/entorno: lo perteneciente y lo no perteneciente.

En la operación de observación, se establece la diferencia entre autorreferencia y heterorreferencia: entre lo que el observador se atribuye a sí mismo y lo que atribuye al sistema observado. Para el caso del sistema de la ciencia, por ejemplo, el sistema distingue entre autorreferencia 
(conceptos) y heterorreferencia (hechos). Se trata, en este caso, de enunciados acerca del sistema (conceptos) y enunciados acerca del entorno del sistema (hechos), pero ambos son enunciados y, por lo tanto, llevados a cabo por el sistema. En otras palabras, el sistema observador distingue internamente entre auto y heterorreferencia, entre lo referido a sí mismo y lo referido a su entorno.

4

La acogida que esta nueva constelación conceptual tuvo en la sociología fue cautelosa. Aunque se había ya presentado el concepto de autopoiesis en congresos de sistemas (Zeleny, 1981), a los que habían asistido también sociólogos, no fue sino hasta que Luhmann lo introdujo en su teoría que la sociología comenzó a aceptarlo. Las disciplinas científicas, incluidas las jóvenes como la sociología, son renuentes a la incorporación de nuevas terminologías y celosas de su autonomía, razón por la cual no es muy bien visto ni fácilmente aceptado que se importe todo un complejo conceptual proveniente de otra disciplina y que, además, recién está siendo presentado en ella.

Hemos visto la elaboración sociológica que hace Luhmann de los conceptos de Maturana. El prestigio intelectual de Luhmann, su conocida capacidad innovadora y la gran complejidad atribuida a su pensamiento hicieron posible que la sociología alemana primero y la internacional después terminaran por aceptar que la autopoiesis y sus conceptos relacionados fueran considerados como pertenecientes al bagaje de la disciplina. La sociología, según Luhmann (1988, p. 292), se encontraba estancada, acumulando datos y volviendo una y otra vez a los clásicos en busca de interpretaciones y reinterpretaciones. Era necesario volver la mirada a los desarrollos que se producían en la teoría de sistemas: en la biología con Maturana y Varela $(1973 ; 1984)$, en la cibernética de segundo orden con Heinz von Foerster (1984), en la lógica formal con Spencer-Brown (1979). 
Con todo ello se podría generar un indispensable cambio de paradigmas que hiciera posible dotar de nuevos esquemas interpretativos a la teoría sociológica. En otras palabras, del mismo modo en que los clásicos de la sociología construyeron un marco conceptual adecuado para entender la sociedad de su época, la complejidad de la sociedad contemporánea requiere de la elaboración de una red de conceptos suficientemente compleja como para dar cuenta de la enorme complejidad de su objeto de estudio. La ciencia de la sociedad, en sus diversas disciplinas estaba desarrollando nuevos marcos interpretativos para explicar el inmenso caudal de datos que año a año se acumulaba. En diversas disciplinas se estaba ya produciendo un cambio de paradigmas que es lo que Luhmann propone para la sociología.

El impacto del trabajo de Luhmann en la sociología ha sido enorme, pero todavía continúa produciéndose. Con posterioridad a la aparición de su obra fundamental, "Die Gesellschaft der Gesellschaft", han seguido publicándose monografías, tales como: "Organisation und Entscheidung" y "Die Religion der Gesellschaft". Otros teóricos han asumido el desafío de investigar distintos aspectos de la vida en la sociedad contemporánea con un marco sistémico en el que los conceptos de autopoiesis y relacionados ocupan un lugar central. Así, las investigaciones de Willke, Baecker, Stichweh, Esposito, Corsi, De Giorgi, por citar sólo algunos, demuestran la vigencia sociológica de conceptos que una vez fueran importados desde la biología de Maturana.

El trabajo de Maturana, por su parte, ha sido leído y discutido fuera de los límites impuestos por la disciplina. Numerosos sociólogos han recurrido a la lectura de los libros fundamentales del autor, interesados en conocer la versión original de los conceptos que han revolucionado la sociología. Otras áreas del quehacer social que también se han visto influenciadas por el pensamiento de Maturana han sido la terapia familiar sistémica y la educación. 
La poderosa adaptación que Luhmann hizo de conceptos provenientes de la biología empírica contribuyó notablemente al refinamiento y precisión conceptual de una teoría destinada a la comprensión de la sociedad moderna. Esta teoría se construyó por espacio de casi treinta años (Luhmann, 1997, p. 11) durante los cuales se fueron agregando conceptos que permitieran distinguir las características de los fenómenos sociales, conservando su particularidad y reduciendo su complejidad, vale decir, contribuyendo a su comprensión selectivamente, pero sin perder características centrales en este proceso de selección.

La teoría sociológica de Niklas Luhmann constituye un impresionante andamio conceptual apropiado para contribuir al conocimiento de la sociedad mundial y de sus procesos. Como toda teoría con pretensiones de universalidad, también ha de encontrarse consigo misma entre sus objetos de estudio. La sociología es - también ella - un fenómeno social y, por lo mismo, parte de su objeto de estudio. La enorme erudición de Luhmann y su disposición hacia la reflexión teórica - que llegó a ser calificada de pasión (Baecker et al., 1987) - le permitió tener un fructífero diálogo interdisciplinario con diversos científicos. La beve reseña que hemos hecho demuestra cómo logró incorporar a la sociología el complejo conceptual de la autopoiesis obteniendo así un modo innovador de reconocer el problema de la autorreferencia como un fenómeno constitutivo de lo social. El diálogo entre Luhmann y Maturana permitió que la sociología contara así con un nuevo instrumental teórico para continuar desarrollando su tarea investigadora.

\section{Referencias}

ALMARAZ, José. La teoría sociológica de Talcott Parsons. Madrid: Centro de Investigaciones sociológicas, 1981.

ASHBY, W. Ross. An introduction to cybernetics. New York: Wiley, 1958. 
BAECKER, Dirk. Information und Risiko in der Markwirtschaft. Frankfurt a.M.: Suhrkamp, 1988.

BAECKER, Dirk. Womit handeln Banken? Frankfurt a.M.: Suhrkamp, 1991.

BAECKER, Dirk. Organisation als System. Frankfurt a.M: Suhrkamp, 1999.

BAECKER, Dirk et al (Hrsg.). Theorie als Passion: Niklas Luhmann zum 60. Geburtstag. Frankfurt a.M: Suhrkamp, 1987.

CORSI, Giancarlo; ESPOSITO, Elena; BARALDI, Claudio. Glosario sobre la teoría social de Niklas Luhmann. México: Anthropos/Universidad Iberoamericana/Iteso, 1996.

DURKHEIM, Emile. De la división del trabajo social. Buenos Aires: Editorial Schapire, 1967.

ELIAS, Norbert. Über der Prozess der Zivilisation. Frankfurt a.M: Suhrkamp, (2 tomos). 7’a edición, 1980.

FOERSTER, Heinz von. Observing Systems. California: Intersystems, 1984.

GARCÍA RUIZ, Pablo; PLAZA DE LA OHZ, Jesús. Talcott Parsons: Elementos para una teoría de la acción social. Pamplona: Publicaciones de la Universidad de Navarra, 2001.

LIPP, Wolfgang. Autopoiesis biologisch, Autopoiesis soziologisch: Wohin führt Luhmanns Paradigmawechsel? Kölner Zeitschrift für Soziologie und Sozialpsychologie, Heft 3, 1987, p. 452-470.

LUHMANN, Niklas. Funktion und Kausalität. Kölner Zeitschrift für Soziologie und Sozialpsychologie 14,1962, p. 617-644.

LUHMANN, Niklas. Moderne Systemtheorien als Form gesamtgesellschaftlicher Analyse. In: Jürgen Habermas y Niklas Luhmann: Theorie der Gesellschaft oder Sozialtechnologie. Frankfurt a.M.: Suhrkamp, 1971, p. 7-24.

LUHMANN, Niklas. Autopoiesis, Handlung und kommunikative Verständigung. Zeitschrift für Soziologie 11, 1982, p. 366-379. 
Sociologias, Porto Alegre, ano 5, no 9, jan/jun 2003, p. 106-140

LUHMANN, Niklas. Soziale Systeme. Frankfurt a.M.: Suhrkamp. Traducción al español: Sistemas sociales. México: Alianza Editorial/Universidad Iberoamericana, 1991.

LUHMANN, Niklas. Neuere Entwicklungen in der Systemtheorie. Merkur, Heft 4, 1988, p. 292-300.

LUHMANN, Niklas. Sistemas Sociales: Lineamientos para una Teoría General. México: Alianza Editorial/Universidad Iberoamericana, 1991.

LUHMANN, Niklas. Die autopoiesis des Bewubtseins. Soziologische Aufklärung 6, Opladen, 1995.

LUHMANN, Niklas. La ciencia de la sociedad. México: Anthropos/Universidad Iberoamericana/Iteso, 1996.

LUHMANN, Niklas. Die Gesellschaft der Gesellschaft. Frankfurt a.M.: Suhrkamp, 1997.

LUHMANN, Niklas. Organisation und Entscheidung. Opladen: Westdeutscher Verlag, 2000a.

LUHMANN, Niklas. Die Religion der Gesellschaft. Frankfurt a.M.: Suhrkamp, $2000 b$.

LUHMANN, Niklas; DE GIORGI, Raffaele. Teoría de la Sociedad, 1a. Ed. Guadalajara, México: Universidad de Guadalajara/Universidad Iberoamericana/Iteso, 1993.

LUHMANN, Niklas; DE GIORGI, Raffaele. Teoría de la sociedad, 2ª . Ed. México: Universidad Iberoamericana/Triana Editores, 1998.

LUHMANN, Niklas; TORRES, Javier. Introducción a la teoría de sistemas. México: Anthropos/Universidad Iberoamericana/Iteso, 1996.

MATURANA, Humberto. Biology of Language: the epistemology of reality. Miller \& Lenneberg (Eds.) Psychology and Biology of Language and Thought. Academic Press, 1978.

MATURANA, Humberto. Erkennen: Die Organisation und Verkörperung von Wirklichkeit. Braunschweig, Wiesbaden: Vieweg \& Sohn, 1982.

MATURANA, Humberto. Comentario manuscrito (no publicado) al libro Sociedad 
y Teoría de Sistemas de Darío Rodríguez y Marcelo Arnold, Santiago de Chile, Enero 1992.

MATURANA, Humberto. I. Fundamentos biológicos de la realidad. In: La realidad: ¿Objetiva o construida? México: Anthropos/Universidad Iberoamericana/Iteso, 1995.

MATURANA, Humberto. II. Fundamentos biológicos del conocimiento. In: La realidad: ¿Objetiva o construida? México: Anthropos/Universidad Iberoamericana/Iteso, 1996.

MATURANA, Humberto; VARELA, Francisco. De máquinas y seres vivos. Santiago: Editorial Universitaria, 1973.

MATURANA, Humberto; VARELA, Francisco. El árbol del conocimiento. Santiago: OEA/Editorial Universitaria, 1984.

PARSONS, Talcott. El sistema social. Madrid: Revista de Occidente, 1966.

RODRÍGUEZ, Darío. Elementos para una comparación de las teorías de Luhmann y Maturana. Estudios Sociales n. 54, 1987.

SCIULLI, David. An interview with Niklas Luhmann, David Sciulli. Theory, Culture \& Society v. 11. London, Thousand Oaks and New Dehli: Sage, 1994.

SPENCER-BROWN, George. Laws of Form. New York: Dutton, 1979.

STICHWEH, Rudolf. Die Weltgesellschaft. Frankfurt a.M.: Suhrkamp, 2000.

WILLKE, Helmut. Systemtheorie. Stuttgart: Gustav Fischer Verlag, 1982.

WILLKE, Helmut. Systemtheorie II: Interventionstheorie. (2 Aufl.) Stuttgart: Lucius \& Lucius, 1996.

WILLKE, Helmut. Systemtheorie III: Steurungstheorie. (2 ${ }^{\circ}$ Aufl.) Stuttgart: Lucius \& Lucius, 1998.

ZELENY, Milan. Autopoiesis: a theory of living organization. Nueva York: North Holland, 1981. 
Sociologias, Porto Alegre, ano 5, no 9, jan/jun 2003, p. 106-140

\section{Resumen}

El artículo ofrece una reseña de la fructífera relación teórica entre Niklas Luhmann y Humberto Maturana. La teoría luhmanniana es un edificio conceptual cuyas dimensiones abarcan la comprensión de todo lo social. Pese al fallecimiento del autor en 1998, todavía hoy se continúan publicando escritos póstumos y la influencia de su pensamiento se extiende paulatinamente a diversos ámbitos de las ciencias sociales. Parte central de este complejo pensamiento se desarrolla con la ayuda de conceptos que Luhmann adaptó de la biología de Humberto Maturana. En este trabajo se explica el modo de esta vinculación y los alcances que tiene para la comprensión de la sociedad globalizada moderna.

Palabras-claves: autopoiesis, emergencia, clausura operacional, comunicación, acoplamiento estructural, autorreferencia. 